\title{
Genotype by environment interaction for adult body weights of shrimp Penaeus vannamei when grown at low and high densities
}

\author{
Ana M. IbARrA ${ }^{1 *}$, Thomas R. FAmUla ${ }^{2}$ \\ ${ }^{1}$ Aquaculture Genetics and Breeding Laboratory, Centro de Investigaciones Biológicas \\ del Noroeste S.C., Mar Bermejo 195, La Paz B.C.S. 23090, Mexico \\ ${ }^{2}$ Department of Animal Science, University of California Davis, 1 Shields Avenue, \\ Davis, CA 95616, USA
}

(Received 14 June 2007; accepted 1st April 2008)

\begin{abstract}
Shrimp is one of few marine species cultured worldwide for which several selective breeding programs are being conducted. One environmental factor that can affect the response to selection in breeding programs is the density at which the shrimp are cultured (low-medium-high). Phenotypic plasticity in the growth response to different densities might be accompanied by a significant genotype by environment interaction, evidenced by a change in heritabilities between environments and by a genetic correlation less than one for a unique trait between environments. Our goal was to understand whether different growth densities affect estimates of those genetic parameters for adult body weight (BW) in the Pacific white shrimp (Penaeus vannamei). BW heritabilities were significantly different between environments, with the largest at high density. These differences resulted from both an increased additive genetic variance and a decreased environmental variance when grown at high density. The genetic correlation between BWs at the two environmental conditions was significantly less than one. Whereas these results might be suggestive for carrying out shrimp selective breeding for BW under high density conditions, further understanding of genetic correlations between growth and reproductive traits within a given environment is necessary, as there are indications of reduced reproductive fitness for shrimp grown at high densities.
\end{abstract}

Litopenaeus / heritability / body weight / density / genotype by environment interaction

\section{INTRODUCTION}

Production of the Pacific white shrimp, Penaeus (Litopenaeus) vannamei, is presently a worldwide activity. This species, whose natural distribution is along the Pacific coast of the western American continent from Mexico to Peru, has been

*Corresponding author: aibarra@cibnor.mx 
introduced into the Atlantic coast from USA to Brazil and into different Asian countries [7]. Between and within different countries, shrimp is cultured in a large variety of environmental and culture conditions and selective breeding programs are being developed. Whereas slight changes in environmental conditions, particularly salinity and temperature, have proven to result in no significant effects on inducing a change in performance rank among genotypes of $P$. vannamei $[8,14]$ and P. japonicus [4], larger changes in grow-out temperature $\left(24-30^{\circ} \mathrm{C}\right)$ and utilization of different stocking densities for $P$. japonicus shrimp culture have indicated that changes in family ranks can occur for growth traits and survival $[5,10]$.

Standard classification of stocking densities for shrimp culture has been rapidly changing, but reported densities range from the minimum stocking densities utilized for extensive pond culture $\left(2-5\right.$ postlarvae $\left.\mathrm{m}^{-2}\right)$ to semi-intensive $\left(8-25 \mathrm{~m}^{-2}\right)$, intensive $\left(25-50 \mathrm{~m}^{-2}\right)$, and super-intensive $\left(>50 \mathrm{~m}^{-2}\right)[11,15]$. For super-intensive culture in raceways, densities up to $400 \mathrm{~m}^{-2}$ have been reported [7]. The challenge will come as we consider the possibility of a genotype by environment interaction (GEI) for production traits. Commercial production systems typically rear animals in environments of less than super-intensive density, whereas many breeding programs, facing issues of biosafety, often turn to super-intensive densities. The presence of GEI will therefore complicate selection programs. Unrecognized changes in the performance rank of families (genotypes) across culture densities resulting from the occurrence of a GEI will lead to a reduced accuracy in breeding value estimation and less than expected genetic gain, although this could depend on the selection scheme being applied [12].

Falconer [6] stated that a unique phenotype "expressed in two environments may be considered to be two genetically correlated character states". As such, not only a significant change in heritability values between environments, but also an estimate of the genetic correlation less than one (i.e., $r_{\mathrm{g}}<1$ ) between a specific phenotype measured in two environments provide evidence of a significant GEI [17].

We present here an analysis of heritabilities of $P$. vannamei shrimp BW at adult, mature age, after growing 102 replicated genotypes (families) at two grow-out density conditions, and an estimate of the genetic correlation for BW between grow-out densities.

\section{MATERIALS AND METHODS}

\subsection{Parental generation}

A two-generation pedigreed structured population of P. vannamei shrimp was used for the analyses. The base generation of the pedigreed population originated from a newly formed breeding line (produced in 2004, with 102 full-sib families) 
was obtained from a commercial shrimp facility in Baja California Sur, Mexico. In 2005, a second generation, also with a total of 102 families, was produced and progeny and parents from these 102 families were used for this study.

The base generation was kept in replicate for security reasons in two grow-out sites: an earthen pond (origin A) and a plastic-liner-covered pond (origin B). In both of these origins, grow-out density at stocking $(1-2 \mathrm{~g})$ was $5-8 \mathrm{shrimp} \cdot \mathrm{m}^{-2}$. Regardless of the same stocking densities, previous observations had indicated that growth in these two environments resulted in different growth rates, possibly associated with different natural productivity at each pond type. Therefore to test for prior possible fixed effects of parent grow-out environments on growth, dams and sires utilized in the production of the progeny generation used in this study were identified as for their 'grow-out origin', their age, and BW obtained after 8 months of grow-out.

\subsection{Production of the families}

All families were produced by artificial insemination using randomly chosen mature individuals. Matings took place over a period of 67 days, from February to April 2005. The only restriction for mating two individuals was if they were known to be related by the pedigree data. A total of 102 families were produced, derived from 79 females and 92 males. Amongst these 102 families, 8 were related to one another through a common sire (i.e., as paternal half-sibs), 20 through a common dam, and 34 with both parents in common. In addition there were 3 families tied by a maternal half-sib trio and 1 family through a paternal half-sib trio.

Larvae production and juvenile rearing of the 102 families were done as described by Perez-Rostro and Ibarra [14]. In short, each family was grown in an individual 100-L tank from nauplii to postlarvae 15-day old (PL15), using an initial stocking density of 100 nauplii $\mathrm{L}^{-1}$, and decreasing it at postlarvae 1-day old (PL1) to $30 \mathrm{PL} 1 \cdot \mathrm{L}^{-1}$. Larvae feeding consisted of a microalgae diet (Chaetoceros calcitrans, Thalassiosira fluviatilis, and Tetraselmis suecica) from nauplii V to advanced zoea III, and the addition of Artemia sp. from zoea III to PL15. The amount of microalgae and the proportion of Artemia sp. added were adjusted daily for each larval stage. After this period of approximately 28 days, each family was transferred and grown in $1 \mathrm{~m}^{2}$ concrete tanks at an initial stocking density of 800 PL15, decreasing it to 400 when juveniles reached an average size of $0.5 \mathrm{~g}$. After 60 days, juveniles reached the size for tagging.

\subsection{Tagging}

A total of 8160 juvenile (1-2 g) shrimp were tagged ( 80 per family). Families were identified by different color elastomers (NMT, Seattle, WA) injected at 
different positions in their abdomen. Half of the individuals tagged per family (40), or a total of 4080 individuals, were then transferred to each one of two grow-out densities in Baja California Sur, Mexico.

\subsection{Test environments}

Half of the tagged shrimp (4080 individuals; $1-2 \mathrm{~g}$ ) were stocked at a low density $\left(5.9 \mathrm{~m}^{-2}\right)$ in a $690 \mathrm{~m}^{2}$ plastic-liner covered bottom pond-type tank at CIBNOR; $5-10 \%$ of the water volume was exchanged daily. Supplemental aeration was provided to maintain oxygen levels above $4 \mathrm{mg} \cdot \mathrm{L}^{-1}$. Density was not purposely adjusted during grow-out, but decreased by natural mortality to 1.8 adult shrimp $\mathrm{m}^{-2}$ at the end of a 9-month grow-out period (June 2005February 2006). The total number of tagged shrimp recovered was 1241, with an estimated survival of $30 \%$ from $1-2 \mathrm{~g}$ to mature age $(\approx 35 \mathrm{~g})$. BWs of all shrimp were collected in February 2006. All families were represented among the survivors, with a mean number per family of 12.2 shrimp (minimum $=$ 1 shrimp per family; maximum $=30$ shrimp per family; mode $=11$ ).

The remaining 4080 tagged individuals were stocked at high density $\left(400 \mathrm{~m}^{-2}\right)$ at the commercial hatchery APBC, using a $308 \mathrm{~m}^{2}$ rectangular raceway tank, and provided with a continuous water flow $\left(15 \mathrm{~L} \cdot \mathrm{s}^{-1}\right)$. To achieve this culture density in the raceway, unmarked same age shrimp were simultaneously grown with the tagged shrimp. Supplemental aeration was also used, maintaining oxygen levels greater than $4 \mathrm{mg} \cdot \mathrm{L}^{-1}$. Density was periodically decreased by natural mortalities, and after 3 months in the raceway, density was adjusted to 50 shrimp $\cdot \mathrm{m}^{-2}$ by randomly transferring out unmarked shrimp. At the end of the study after a grow-out period of 10 months (June 2005-March 2006), the density in the raceway was 38 adult shrimp $\mathrm{m}^{-2}$. The total number of tagged shrimp recovered was 1064, with an estimated survival of $26 \%$ from 1-2 g to mature age $(\approx 35 \mathrm{~g})$. BWs of all shrimp were collected in March 2006. All families were represented among the survivors, with a mean number per family of 10.5 shrimp (minimum $=1$ shrimp per family; maximum $=24$ shrimp per family; mode $=10$ ).

To test for differences in survival patterns of the families when grown at each of the two environments, a correlation analysis was done between the numbers of shrimp surviving per family at each environment. The resulting correlation was positive and significant $(r=0.27, P=0.007)$. Though significantly different from zero, this correlation suggests a weak (though positive) relationship between survival patterns in the two densities, which is the cause for further concern about the impact of GEI in the construction of breeding programs. 


\subsection{Feeding}

Feeding in both cases was done on demand, using a commercial pellet $(40 \%$ protein) and tray-feeding. For the low density tank, food was added to the trays three times a day and for the high density raceway every $2 \mathrm{~h}$.

\subsection{Statistical and genetic parameter analyses}

\subsubsection{Model evaluation}

Means and standard deviation (SD) of BWs unadjusted for age for each grow-out density and sex were estimated using STATISTICA v.5 software.

A linear model was used to evaluate the significance of fixed effects and covariates utilizing SOLAR (Sequential Oligogenic Linkage Analysis Routines; Version 4.0.5 for Linux, downloaded on September 8, 2006 from http://www.sfbr.org/solar/) software [1]. Age and age-squared were used as covariates, and density, sex, origin, and their interactions were used for fixed effects. Neither dam nor sire origin (see Sect. 2 for origin A or B) nor their interaction with density were significant $(P>0.05)$ and therefore they were excluded from all further analyses.

\subsubsection{Heritabilities}

For the objective of estimating the heritability of BW across the two densities, a single trait linear animal model was used. This model included terms for the fixed effects; sex of shrimp, density class, a sex by density interaction and covariates for age and age-squared, and an animal genetic effect. Algebraically, the model for the $\mathrm{BW}$ of the $j$ th animal $\left(\mathbf{y}_{j}\right)$ was:

$$
\mathbf{y}_{j}=\mu+\boldsymbol{\beta}^{\prime} \mathbf{x}_{j}+\mathbf{g}_{j}+\mathbf{e}_{j}
$$

where $\mu$ is a constant common to all animals, $\mathbf{x}_{j}$ is the vector of covariates and fixed effects (i.e., age, sex, density, sex by density) for the $j$ th individual, $\boldsymbol{\beta}$ is the vector of corresponding unknown fixed effects, $\mathbf{g}_{j}$ is modeled as the additive genetic, polygenic contribution to $\mathrm{BW}$, and $\mathbf{e}_{j}$ is the corresponding unknown residual. The distribution of the unobservable additive genetic contribution and the residual were assumed to be multivariate normal, and independent of one another. In addition, defining $\mathbf{g}$ as the vector of additive genetic values for all individuals in the pedigree, we assume that $\operatorname{Var}[\mathbf{g}]=\mathbf{A} \sigma_{\mathrm{g}}^{2}$, where $\mathbf{A}$ is the matrix of numerator relationships and $\sigma_{\mathrm{g}}^{2}$ is the unknown additive genetic variance. In a similar fashion, defining $\mathbf{e}$ as the vector of residuals, we assume that $\operatorname{Var}[\mathbf{e}]=\mathbf{I} \sigma_{\mathrm{e}}^{2}$, where $\mathbf{I}$ is an identity 
matrix and $\sigma_{\mathrm{e}}^{2}$ is the unknown residual variance. It is important to note that, because of the mating system, $\mathbf{g}$ may contain effects beyond the additive genetic contribution to BW.

\subsubsection{Genetic correlations}

Estimation of the genetic correlation across environments is a straightforward and commonly used technique for the identification of GEI (e.g., Via and Hawthorne [18]). Expansion of the above single trait model to a multiple trait model across environments is easily accomplished. That is, we consider BW in the low density environment to be a different trait from BW in the high density environment, allowing for the establishment of a multiple trait model. The model for BW across the two density classes $\left(\mathbf{y}_{j 1}\right.$ or $\left.\mathbf{y}_{i h}\right)$, the high density class (subscript ' $h$ ') and low density class (subscript ' $\mathrm{l}$ '), between two shrimp $i$, nested in the high density environment and shrimp $j$, nested in the low density environment, was:

$$
\begin{aligned}
& \mathbf{y}_{j 1}=\mu_{1}+\boldsymbol{\beta}_{1}^{\prime} \mathbf{x}_{j 1}+\mathbf{g}_{j 1}+\mathbf{e}_{j 1} \\
& \mathbf{y}_{i \mathrm{~h}}=\mu_{\mathrm{h}}+\boldsymbol{\beta}_{\mathrm{h}}^{\prime} \mathbf{x}_{i \mathrm{~h}}+\mathbf{g}_{i \mathrm{~h}}+\mathbf{e}_{i \mathrm{~h}}
\end{aligned}
$$

where $\mu_{1}\left(\mu_{\mathrm{h}}\right)$ is a constant common to all animals in the low (high) density environment, $\mathbf{x}_{j 1}\left(\mathbf{x}_{i \mathrm{~h}}\right)$ is the vector of covariates and fixed effects (i.e., age, sex) for the $j$ th individual (ith individual) in the low (high) density environment, $\boldsymbol{\beta}_{1}\left(\boldsymbol{\beta}_{\mathrm{h}}\right)$ is the vector of corresponding unknown fixed effects for the low (high) density environment, $\mathbf{g}_{j 1}\left(\mathbf{g}_{i \mathrm{~h}}\right)$ is modeled as the additive genetic, polygenic contribution to $\mathrm{BW}$ in the low (high) density environment, and $\mathbf{e}_{j 1}\left(\mathbf{e}_{i h}\right)$ is the corresponding unknown residual for the low (high) density environment. Moreover, defining the $\mathbf{g}_{1}\left(\mathbf{g}_{\mathrm{h}}\right)$ as vectors of additive genetic values for all individuals in the low (high) density environments,

$$
\operatorname{Var}\left[\begin{array}{l}
\mathbf{g}_{1} \\
\mathbf{g}_{\mathrm{h}}
\end{array}\right]=\mathbf{G}=\mathbf{G}_{0} \otimes \mathbf{A}=\left[\begin{array}{cc}
\sigma_{1}^{2} & \sigma_{\mathrm{lh}} \\
\sigma_{\mathrm{lh}} & \sigma_{\mathrm{h}}^{2}
\end{array}\right] \otimes \mathbf{A}
$$

where $\sigma_{1}^{2}$ is the additive genetic variance for shrimp in the low density environment, $\sigma_{\mathrm{h}}^{2}$ is the additive genetic variance for shrimp in the high density environment, $\sigma_{\mathrm{lh}}$ is the additive genetic covariance across the low and high density environments, $\mathbf{A}$ is the numerator relationship matrix among all shrimp, and $\otimes$ is the symbol for a direct product. In addition, define $\mathbf{e}_{1}$ and 
Table I. Means for BW (and SD) of P. vannamei male and female adult shrimp when grown at low vs. high stocking densities.

\begin{tabular}{lrccc}
\hline $\begin{array}{l}\text { Grown at } \\
\text { Sex }\end{array}$ & $N$ & $\begin{array}{c}\text { Adults mean } \\
\text { body weight }(\mathrm{g})(\mathrm{SD})\end{array}$ & $\begin{array}{c}\text { Coefficient of } \\
\text { variation }(\%)\end{array}$ & $\begin{array}{c}\text { Range in } \\
\text { weights }(\mathrm{g})\end{array}$ \\
\hline $\begin{array}{l}\text { Low density } \\
\text { Males }\end{array}$ & 1241 & & & \\
$\quad 707$ & $33.55(4.07)$ & 12.14 & $18.9-46.1$ \\
$\quad$ Females & 534 & $38.46(5.76)$ & 14.99 & $24.8-56.3$ \\
High density & 1064 & & & \\
$\quad$ Males & 539 & $31.70(3.65)$ & 12.14 & $20.0-41.4$ \\
Females & 525 & $34.77(5.15)$ & 14.81 & $21.3-51.6$ \\
\hline
\end{tabular}

Coefficient of variation and ranges in weights are included.

$\mathbf{e}_{\mathrm{h}}$ as the vectors of residuals for the low and high density BWs, respectively, then

$$
\operatorname{Var}\left[\begin{array}{l}
\mathbf{e}_{1} \\
\mathbf{e}_{\mathrm{h}}
\end{array}\right]=\mathbf{R}=\mathbf{R}_{0} \otimes \mathbf{I}=\left[\begin{array}{cc}
\sigma_{\mathrm{el}}^{2} & 0 \\
0 & \sigma_{\mathrm{eh}}^{2}
\end{array}\right] \otimes \mathbf{I}
$$

where $\sigma_{\mathrm{el}}^{2}$ is the residual variance for shrimp in the low density environment and $\sigma_{\text {eh }}^{2}$ is the residual variance for shrimp in the high density environment. Note that the residual covariance is defined as zero. Because individual shrimp are nested within a density class, no animal can be observed in both environmental classes (i.e., traits). Accordingly, the environmental correlation cannot be estimated from the present data. Estimates of the unknown variances and covariances (along with the associated heritabilities and genetic correlation) were implemented through the public domain computer program MTDFREML [3].

\section{RESULTS}

Means of BWs by sex and density are presented in Table I.

The fixed effects of dam and sire origin, and their interaction with density were not significant $(P>0.05)$. The covariate age (and age-squared) and the fixed effects of sex and density, as well as their interaction, were all significant $(P<0.001)$ and therefore included in all further analyses. Specifically, the difference between females and males was estimated as $2.91 \mathrm{~g}( \pm 0.23)$ at the high density and $4.39 \mathrm{~g}( \pm 0.24)$ at the low density, using estimates of the sex effects in the mixed linear model.

The heritability of shrimp BW at adult age in the combined environments using the reduced linear model that excluded only origin (i.e., leaving sex and 
Table II. (a) Heritabilities (on diagonal) and genetic correlation (above the diagonal) for BW across density class with standard error. Variance (on diagonal) and covariance (above the diagonal) components of (b) genetic, and (c) environmental contribution to growth of $P$. vannamei shrimp across two density classes.

\begin{tabular}{lll}
\hline & Low density & High density \\
\hline (a) Heritability and genetic correlation & & \\
Low density & $0.35(0.05)$ & $0.54(0.12)$ \\
High density & & $0.61(0.08)$ \\
(b) Genetic (co)variances & & \\
Low density & 7.61 & 5.08 \\
$\quad$ High density & & 11.84 \\
(c) Environmental (co)variances & 14.26 & 0.0 \\
Low density & & 7.58 \\
High density & & \\
\hline
\end{tabular}

age in the model) as a fixed effect was $h^{2}=0.46 \pm 0.065(P<0.001)$. Table II presents estimates of the heritability for adult shrimp BW estimated separately for each density class. In each case, the values of heritability were significantly different from zero, as indicated by their standard errors. The different heritabilities across environments were caused by different additive genetic and environmental variances occurring at each environment for growth: a larger environmental variance and smaller additive genetic variance were observed for the low density environment.

Our estimate of the genetic correlation between BWs recorded in the two density test environments (Tab. II) was $0.54 \pm 0.12$, providing strong evidence of considerable GEI for growth in these two density classes. Building crude 95\% confidence intervals (i.e., twice the standard error) around the correlation estimate, we further get the indication that the genetic correlation is less than one.

\section{DISCUSSION}

Previous studies have found indications of a GEI for growth of $P$. japonicus when just a few families were grown at different stocking densities $[5,10]$ and for growth, biomass, and survival when grown at extreme temperatures [4]. Our results confirmed those previous findings when evaluated in P. vannamei. It is known that a genetic correlation less than one for a specific trait when measured in two environments is an indication of a significant GEI [18]. The observed genetic correlation provided strong evidence that the genes involved 
in shrimp growth at a low density environment are either not the same suite of loci, interact differently, or are differently expressed when shrimp is grown in a high density environment.

Further evidence for stating that different genes were involved in growth in the two environments came from the estimated additive genetic and environmental variances for each density in the present study, which resulted in different heritability estimates. It is well known that a change in environmental conditions can affect the average expression of traits, the amount of genetic and environmental variance of the traits, and consequently the heritability of the traits [9]. Whereas a change in heritabilities in stressful environments could be a function of different environmental variances across environments, with the additive genetic variance being constant, that was not the case for the present study. Not only was the environmental variance decreased when shrimp were grown at a high density, but also the additive genetic variance was increased. This was in agreement with previous findings in different species for which stressful conditions generally increase genetic variation [9].

Based on these results, it might be thought that selection for shrimp improvement should be done after growing it at high densities. However, before selection for BW is practiced in this type of environment, a further understanding of the effects on genetic correlations of BW with other traits within this type of environment is necessary. In particular, the genetic correlation between BW and reproductive capacity (number of spawns, days to first spawn, fecundity, larval survival, etc.) has to be estimated. It is known that genetic correlations among traits can be environment-dependent and can be changed when environmental conditions are modified [16]. Early life environmental conditions appear to have a significant effect on the maturation condition of sub-adult $P$. vannamei, with poor conditions resulting in no development of vitellogenic oocytes [13], whereas when grown in normal conditions vitellogenic oocytes are already developing in sub-adults [2]. The previous studies indicate that any existing (and beneficial) genetic correlation between BW and reproductive capacity might change at high densities, and that further research is required before proposing selective breeding at high densities. This conclusion also derives from observations in this study: inasmuch as the present study was not aimed at evaluating reproductive performance of shrimp grown at each density, data obtained for females selected from the top 10\% BW distribution during spawning indicate a diminished reproductive capacity (measured as egg vitellin concentration, number of spawns, and number of viable nauplii) of females when grown under high density condition when compared with those grown under low density condition (A.M. Ibarra, unpublished results). 


\section{ACKNOWLEDGEMENTS}

This research was supported by CIBNOR and by the commercial shrimp company Acuacultores de la Peninsula de B.C., S.A. de C.V. through his General Director, Jaime J. Malagamba-Ansotegui. We thank Ximena Malagamba and Juan M. Mackliz for technical support and data collection.

\section{REFERENCES}

[1] Almasy L., Blangero J., Multipoint quantitative-trait linkage analysis in general pedigrees, Am. J. Hum. Genet. 62 (1998) 1198-1211.

[2] Arcos F.G., Racotta I.S., Palacios E., Ibarra A.M., Ovary development at the onset of gametogenesis is genetically determined and correlated with reproductive traits at maturity in shrimp Litopenaeus (Penaeus) vannamei, Mar. Biol. 148 (2005) 339-346.

[3] Boldman K.G., Kriese L.A., van Vleck L.D., van Tassell C.P., Kachman S.D., A manual for use of MTDFREML. A set of programs to obtain estimates of variances and covariances [Draft], US Department of Agriculture, Agricultural Research Service, 1995.

[4] Coman G.J., Crocos P.J., Preston N.P., Fielder D., The effects of temperature on the growth, survival and biomass of different families of juvenile Penaeus japonicus Bate, Aquaculture 214 (2002) 185-199.

[5] Coman G.J., Crocos P.J., Preston N.P., Fielder D., The effects of density on the growth and survival of different families of juvenile Penaeus japonicus Bate, Aquaculture 229 (2004) 215-223.

[6] Falconer D.S., The problem of environment and selection, Am. Nat. 86 (1952) 293-298.

[7] Funge-Smith S., Briggs M., The introduction of Penaeus vannamei and P. stylirostris into the Asia-Pacific region. Case studies, in: Bartley D.M., Bhujel R.C., Funge-Smith S., Olin P.G., Phillips M.J. (Eds.), International Mechanisms for the Control and Responsible use of Alien Species in Aquatic Ecosystems, Report of an Ad Hoc Expert Consultation, FAO, Rome, 2005, pp. 149-167, http://library.enaca.org/NACA-Publications/alienspecies/a0113e.pdf [consulted: May 2007].

[8] Gitterle T., Rye M., Salte R., Cock J., Johansen H., Lozano C., Suárez J.A., Gjerde B., Genetic (co)variation in harvest body weight and survival in Penaeus (Litopenaeus) vannamei under standard commercial conditions, Aquaculture 243 (2005) 83-92.

[9] Hoffmann A.A., Parsons P.A., Evolutionary genetics and environmental stress, 1st edn., Oxford University Press, Oxford, 1991.

[10] Jerry D.R., Preston N.P., Crocos P.J., Keys S., Meadows J.R.S., Li Y., Application of DNA parentage analyses for determining relative growth rates of 
Penaeus japonicus families reared in commercial ponds, Aquaculture 254 (2006) 171-181.

[11] Kunhimohamed A.B., Support to special plan for prawn and shrimp farming, Myanmar, Technical Cooperation Programme, FAO, Bangkok. TCP/MYA/4554, Field Document, 1998, http://www.fao.org/docrep/field/382903.htm\#1.2\%20 terms $\% 20$ of $\% 20$ reference [consulted: April 25, 2007].

[12] Mulder H.A., Bijma P., Effects of genotype $\times$ environment interaction on genetic gain in breeding programs, J. Anim. Sci. 83 (2005) 49-61.

[13] Parnes S., Mills E., Sagall C., Raviv S., Davis C., Sagi A., Reproductive readiness of the shrimp Litopenaeus vannamei grown in a brackish water system, Aquaculture 236 (2004) 593-606.

[14] Perez-Rostro C.I., Ibarra A.M., Heritabilities and genetic correlations of size traits at harvest size in sexually dimorphic Pacific white shrimp (Litopenaeus vannamei) grown in two environments, Aquacult. Res. 34 (2003) 1-7.

[15] Raux P., Bailly D., Literature review on world shrimp farming, in: Individual Partner Report for the Project: Policy Research for Sustainable Shrimp Farming in Asia, European Commission INCODEV, Project No. IC4-2001-10042, CEMARE University of Portsmouth UK and CEDEM, Brest, France, 2002, 46 p.

[16] Sgrò C.M., Hoffmann A.A., Genetic correlations, tradeoffs and environmental variation (review), Heredity 93 (2004) 241-248.

[17] Via S., Genetic constraints on the evolution of phenotypic plasticity, in: Loeschcke V. (Ed.), Genetic Constraints on Adaptive Evolution, SpringerVerlag, Berlin, 1987, pp. 47-71.

[18] Via S., Hawthorne D.J., Back to the future: genetic correlations, adaptation and speciation, Genetica 123 (2005) 147-156. 